\title{
AVAILABILITY AND UPTAKE OF NITROGEN AND POTASSIUM AS AFFECTED BY INORGANIC FERTILIZERS IN FLOODED RICE SOILS \\ El-Hamdi, Kh. H. ${ }^{*}$; S. A. Ghanem ${ }^{\star *}$ and I. M. Hashem ${ }^{\star *}$ \\ * Soils Dept., Fac. Agric., Mansoura Univ., Egypt. \\ ** Rice Research and Training Center, Sakha, Kafr El-Sheikh
}

\begin{abstract}
Effect of nitrogen $(\mathrm{N})$ and potassium $(\mathrm{K})$ fertilizers application on the uptake and availability of nitrogen and potassium was studied at the Farm of Rice Research and Training Center at Sakha Kafr El-sheikh during 2007 and 2008 seasons. The highest values of available $\mathrm{NH}_{4}-\mathrm{N}$ in the soil were recorded at 30 DAT under $92 \mathrm{~kg} \mathrm{~N}$ $\mathrm{fed}^{-1}$ with application full dose of potassium before transplanting $(50 \mathrm{Kg}$ potassium sulphate. $\mathrm{fed}^{-1}$ ). Data showed also that highest values of available soil $\mathrm{K}$ were obtained at 30 DAT when rice plants were fertilized with $92 \mathrm{~kg} \mathrm{~N} \mathrm{fed}^{-1}$ combined with application of full dose of potassium before transplanting in 2007 and 2008 seasons, respectively. The highest values of nitrogen and potassium uptake were recorded at 30 days after transplanting (DAT) when rice plants were fertilized with $92 \mathrm{~kg} \mathrm{~N}$ fed ${ }^{1}$ combined with application of potassium full dose $\left(50 \mathrm{~kg}\right.$ potassium sulphate fed ${ }^{-1}$ ) before flooding at both seasons of study. Data showed also that maximum nitrogen uptake values were found when $92 \mathrm{~kg} \mathrm{~N}^{-1}$ fed was combined with application of potassium (half dose before transplanting + spray with $2 \% \mathrm{~K}_{2} \mathrm{O}(4 \mathrm{~kg}$ potassium sulphate) at panicle initiation stage + spray with $2 \% \mathrm{~K} 2 \mathrm{O}$ (4kg potassium sulphate) at flowering stages). The $\mathrm{N}$ uptake increased significantly at 50,70 and 95 days after transplanting (DAT) in both seasons of study. Data showed also that application of nitrogen at the rate of $92 \mathrm{~kg} \mathrm{~N}$ fed $^{-1}$ in combined with application half dose of recommended amount potassium before transplanting + spray with $2 \% \mathrm{~K}_{2} \mathrm{O}$ at panicle initiation and flowering stages gave the highest grain yield in 2007 and 2008 seasons, respectively.
\end{abstract}

Keywords: Availability, uptake, nitrogen, potassium and rice flooded soil.

\section{INTRODUCTION}

The crop is generally fertilized by farmers either with nitrogen or with nitrogen and phosphorus only, through potassium is equally important as it stabilizes yield and it's a quality nutrient. Nitrogen is considered the limiting factor for rice growth. Secondary minerals may affect the availability of $\mathrm{K}$ irreversibly fixed in the interlayer and wedge sites of soil clay and is rendered unavailable to growing plants (Arshad and Akram, 1999). During weathering, physical disintegration of mica into clay size fractions resulted in replacement and release of interlayer $\mathrm{K}$ by more hydrated cations $\left(\mathrm{Ca}^{2+}, \mathrm{Mg}^{2+}\right.$, or $\left.\mathrm{Na}^{+}\right)$. Evidence of particle size reduction has been found in alluvial soils of Gujranwala, Lyallpur, and Burhan series (Akhtar, 1989).

The amount of fixed $\mathrm{K}$ in soil increases with added $\mathrm{K}$, whereas the present $\mathrm{K}$ fixed relative to total added $\mathrm{K}$ decreases (Bouabid et al., 1991). Fixation of $\mathrm{K}$ fertilizers may affects its recovery by crops. Application of potassium along with nitrogen has become very necessary due to intensified agriculture with high yielding varieties. Introduction of hybrid rice is an important step towards augmentation of rice yield. Hybrid rice is highly 


\section{El-Hamdi, Kh. H. et al.}

fertilizer responsive, but there is a shortage in hybrid rice nutrition. So, adequate fertilization and combination at real time in a proper manner is essential to achieve potentially yield of hybrid rice.

The present work was designed to evaluate the combined effects of different levels of $\mathrm{N}$ and different methods of $\mathrm{K}$ application on:

1- Availability of ammonium and potassium in flooded rice soils.

2- Uptake of nitrogen and potassium in rice.

3- Grain yield of hybrid rice

\section{MATERIALS AND METHODS}

A field experiment was carried out on the transplanted rice system at the Experimental Farm of the Rice Research and Training Center (RRTC), Sakha, Kafr El-Sheikh, Egypt, during the two growing summer seasons of 2007 and 2008 to investigate the effect of nitrogen levels and different methods of potassium application on the nutrient uptake, availability of some nutrients and grain yield of hybrid rice.

\section{Soil Analyses:}

Soil sample was taken before cultivation crushed then grinned to pass through $2 \mathrm{~mm}$ sieve, some physical and chemical analysis were determined according to Cottenie et al, (1982) and Page et al, (1982). The results are presented in Table 1.

\section{Experimental Design:}

Experimental design was laid out in a split plot design with four replications. The main plots were devoted to the three nitrogen levels in urea form,

1) Zero nitrogen $\left(\mathrm{N}_{1}\right)$.

2) $69 \mathrm{~kg} \mathrm{~N} \mathrm{fed}^{-1}\left(\mathrm{~N}_{2}\right)$.

3) $92 \mathrm{~kg} \mathrm{~N} \mathrm{fed}^{-1}\left(\mathrm{~N}_{3}\right)$.

Where the sub plots occupied by the eight methods of potassium application at the rate of $24 \mathrm{~kg} \mathrm{~K}_{2} \mathrm{O}\left(\mathrm{fed}^{-1}\right)$ in potassium sulphate form (50 kg fed $\left.{ }^{-1}\right)$,

1) Zero potassium $\left(K_{1}\right)$.

2) All amount was applied as basal (B) incorporated into the soil surface $\left(\mathrm{K}_{2}\right)$.

3) Half dose of potassium fertilizer was applied as basal and incorporated into the soil surface + spray with $2 \% \mathrm{~K}_{2} \mathrm{O}$ ( $4 \mathrm{~kg}$ potassium sulphate) was applied at panicle initiation $\left(\mathrm{PI}\left(\mathrm{K}_{3}\right)\right.$.

4) Half dose of potassium fertilizer was applied as basal and incorporated into the soil surface + spray with $2 \% \mathrm{~K}_{2} \mathrm{O}$ (4kg potassium sulphate) was applied at flowering $(\mathrm{F})\left(\mathrm{K}_{4}\right)$.

5) Half dose of potassium fertilizer was applied as basal and incorporated into the soil surface + spray with $2 \% \mathrm{~K}_{2} \mathrm{O}$ was applied at panicle initiation $(\mathrm{PI})$ and flowering $(\mathrm{F})\left(\mathrm{K}_{5}\right)$.

6) Spray with $2 \% \mathrm{~K} 2 \mathrm{O}$ (4kg potassium sulphate) was applied at panicle initiation $(\mathrm{PI})\left(\mathrm{K}_{6}\right)$.

7) Spray with $2 \% \mathrm{~K} 2 \mathrm{O}$ (4kg potassium sulphate) was applied at flowering $(\mathrm{F})\left(\mathrm{K}_{7}\right)$.

8) Spray with $2 \% \mathrm{~K} 2 \mathrm{O}(4 \mathrm{~kg}$ potassium sulphate) was applied at panicle initiation $(\mathrm{PI})$ and flowering $(\mathrm{F})\left(\mathrm{K}_{8}\right)$. 
Table 1: Some physical and chemical analysis of soil used

\begin{tabular}{|c|c|c|}
\hline Soil properties & 2007 & 2008 \\
\hline - $\quad$ Clay\% & 55.9 & 56.0 \\
\hline - $\quad$ Silt $\%$ & 31.5 & 32.0 \\
\hline - $\quad$ Sand\% & 12.6 & 12.0 \\
\hline - $\quad$ Texture & Clayey & Clayey \\
\hline - $\quad$ Total nitrogen, $\mathrm{mg} \mathrm{kg}^{-1}$ & 520 & 600 \\
\hline - $\quad$ Available $\mathrm{P}, \mathrm{mg} \mathrm{kg}^{-1}\left(0.5 \mathrm{M} \mathrm{NaHCO}_{3}\right)$ & 13.0 & 16.0 \\
\hline - $\quad$ Available ammonium ( $\mathrm{mg} \mathrm{kg}^{-1}$ ) & 17.2 & 18.8 \\
\hline - $\quad$ Nitrate concentration $\left(\mathrm{mg} \mathrm{kg}^{-1}\right)$ & 13.0 & 14.1 \\
\hline - $\quad$ Available Potassium (mg kg $\left.{ }^{-1}\right)^{\prime}$ & 310 & 325 \\
\hline - $\quad \mathrm{pH}(1: 2.5$ soil suspension) & 8.1 & 8.2 \\
\hline $\begin{array}{ll}\text { - } & \text { EC dS.m } \mathrm{m}^{-1} \text { (soil paste) } \\
\text { - } & \text { Soluble cations, meq. } \mathrm{L}^{-1} \text { (soil paste): }\end{array}$ & 2.1 & 2.3 \\
\hline - $\mathrm{Ca}^{++}$ & 5.2 & 5.4 \\
\hline$-\mathrm{Mg}^{++}$ & 2.0 & 2.1 \\
\hline$-\mathrm{K}^{+}$ & 0.5 & 0.4 \\
\hline$-\mathrm{Na}^{+}$ & 12.1 & 12.3 \\
\hline - $\quad$ Soluble anions, meq..$^{-1}$ : & & \\
\hline $\mathrm{CO}_{3}^{--}$ & 0.00 & 0.00 \\
\hline $\mathrm{HCO}_{3}^{-}$ & 3.6 & 3.9 \\
\hline - $\mathrm{Cl}^{-}$ & 14.9 & 15.1 \\
\hline$-\mathrm{SO}_{4}^{-}$ & 1.30 & 1.2 \\
\hline $\begin{array}{ll}- & \text { Available micronutrients } \mathbf{~ m g ~ k g} \\
- & \mathrm{Fe}^{++}\end{array}$ & 6.0 & 6.2 \\
\hline$-\quad \mathrm{Zn}^{++}$ & 0.9 & 1.1 \\
\hline$-\quad \mathrm{Mn}^{++}$ & 3.5 & 3.6 \\
\hline
\end{tabular}

\section{Plant samples:}

During the growth stage, plant samples were taken at 30,50,70 and 95 days after transplanting (DAT). All plant samples were placed in paper bags and oven dry at $70^{\circ} \mathrm{C}$ for 48 hours. Dried samples were ground to powder and digested according to the method of Chapman and Pratt (1961), prior to chemical analysis as follows.

\section{Plant analysis:}

- Nitrogen content was determined using Orange-G dye colorimetric method according to Hafez and Mikkelson (1981).

- Potassium was determined by the flame photometer as described by Jackson (1967).

- Soil sampling:

Soil samples were collected from each treatment $(0-20 \mathrm{Cm}$ depth) four times at 30,50, 70 and 95 days after transplanting (DAT). The soil samples were homogenized and frozen directly after collection to prevent microbial activity. After thawing samples were immediately extracted to determine the nutrient elements. Available ammonium and potassium in the soil sample were determined according to Cottenie et al., (1982). 


\section{RESULTS AND DISCUSSION}

1- Effect of nitrogen and potassium fertilization on nutrients availability: 1.1- Ammonium availability:

Available $\mathrm{NH}_{4}-\mathrm{N}$ at different periods throughout rice growing seasons as affected by nitrogen levels and methods of potassium application are presented in Tables 2 and 3.

Data show that available $\mathrm{NH}^{+}{ }_{4}$ sharply increased after flooding reached its high peak at 30 DAT in 2007 and 2008 seasons, and then declined to the minimum by 95 DAT. De Datta (1981) stated that the greater part of N mineralized during rice season appears as ammonium with in two weeks after submergence. It is worthy to mention that the other doses of $\mathrm{N}$ fertilizer at mid tillering, panicle initiation and flowering stages did not increase the mean values of $\mathrm{NH}_{4}$. This could be attributed to the rapid absorption of this ion by rice roots for tillers production and initiation of panicles. These results in agreement with those reported by Young et al. (2005).

Table 2: $\mathrm{NH}_{4}$ availability in soil $\left(\mathrm{mg} \mathrm{kg}^{-1}\right)$ as affected by nitrogen levels and potassium application during 2007 seasons.

\begin{tabular}{|c|c|c|c|c|c|c|c|c|c|}
\hline \multirow{3}{*}{ N Treatments } & \multicolumn{9}{|c|}{ K- Treatments } \\
\hline & \multicolumn{9}{|c|}{30 DAT } \\
\hline & K1 & K2 & K3 & K4 & K5 & K6 & K7 & K8 & Mean \\
\hline N1 & 28.51 & 40.10 & 34.85 & 35.75 & 35.82 & 27.70 & 26.90 & 28.95 & 32.32 \\
\hline N2 & 41.90 & 51.65 & 45.85 & 46.20 & 46.05 & 40.57 & 40.45 & 40.15 & 44.09 \\
\hline N3 & 60.65 & 67.70 & 63.50 & 63.20 & 63.00 & 60.00 & 61.05 & 61.20 & 62.50 \\
\hline \multirow[t]{2}{*}{ Mean } & 43.68 & 53.15 & 48.06 & 48.38 & 48.29 & 42.75 & 42.76 & 43.43 & 46.30 \\
\hline & \multicolumn{9}{|c|}{50 DAT } \\
\hline N1 & 20.45 & 30.45 & 25.40 & 26.09 & 25.15 & 20.00 & 21.10 & 20.49 & 23.64 \\
\hline N2 & 33.60 & 40.00 & 35.90 & 36.23 & 35.00 & 33.00 & 32.90 & 33.85 & 35.06 \\
\hline N3 & 43.95 & 50.40 & 47.95 & 47.10 & 46.30 & 43.55 & 43.00 & 44.05 & 45.78 \\
\hline \multirow[t]{2}{*}{ Mean } & 32.66 & 40.28 & 36.41 & 36.47 & 35.48 & 32.18 & 32.33 & 32.79 & 34.82 \\
\hline & \multicolumn{9}{|c|}{70 DAT } \\
\hline N1 & 17.35 & 24.00 & 21.30 & 21.60 & 20.80 & 16.95 & 17.05 & 17.25 & 19.53 \\
\hline N2 & 26.00 & 30.40 & 30.00 & 29.15 & 29.50 & 27.00 & 26.30 & 26.90 & 28.15 \\
\hline N3 & 32.70 & 39.99 & 37.50 & 36.95 & 37.00 & 33.00 & 32.80 & 33.60 & 35.44 \\
\hline \multirow[t]{2}{*}{ Mean } & 25.35 & 31.46 & 29.60 & 29.23 & 29.10 & 25.65 & 25.38 & 25.91 & 27.70 \\
\hline & \multicolumn{9}{|c|}{95 DAT } \\
\hline N1 & 14.00 & 17.70 & 16.66 & 16.95 & 15.80 & 14.90 & 15.00 & 14.60 & 15.72 \\
\hline N2 & 20.10 & 23.30 & 24.00 & 24.50 & 23.00 & 20.00 & 20.80 & 19.90 & 21.95 \\
\hline N3 & 24.00 & 29.50 & 30.20 & 31.10 & 29.00 & 26.25 & 25.65 & 25.00 & 27.58 \\
\hline Mean & 19.60 & 23.50 & 23.62 & 24.18 & 22.60 & 20.38 & 20.48 & 19.83 & 21.75 \\
\hline 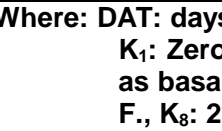 & & & & & & & & & \\
\hline
\end{tabular}


Table 3: $\mathrm{NH}_{4}$ availability in soil $\left(\mathrm{mg} \mathrm{kg}^{-1}\right)$ as affected by nitrogen levels and potassium application during 2008 seasons.

\begin{tabular}{|c|c|c|c|c|c|c|c|c|c|}
\hline \multirow{3}{*}{$\mathbf{N}$ Treatments } & \multirow{2}{*}{\multicolumn{9}{|c|}{ K- Treatments }} \\
\hline & \multicolumn{4}{|c|}{30 DAT } & & & & & \\
\hline & K1 & K2 & K3 & K4 & K5 & K6 & K7 & K8 & Mean \\
\hline N1 & 32.20 & 43.33 & 39.00 & 38.00 & 38.50 & 32.80 & 33.00 & 33.15 & 36.24 \\
\hline N2 & 45.23 & 54.90 & 50.10 & 50.00 & 51.00 & 44.10 & 45.00 & 45.20 & 48.19 \\
\hline N3 & 61.40 & 69.00 & 65.00 & 65.30 & 65.60 & 60.34 & 61.00 & 61.90 & 63.56 \\
\hline \multirow[t]{2}{*}{ Mean } & 46.27 & 55.74 & 51.36 & 50.76 & 51.70 & 45.74 & 46.33 & 46.75 & 49.33 \\
\hline & \multicolumn{9}{|c|}{50 DAT } \\
\hline N1 & 24.95 & 32.50 & 28.75 & 29.15 & 28.45 & 24.60 & 23.70 & 24.00 & 27.01 \\
\hline N2 & 35.33 & 42.45 & 38.82 & 38.00 & 39.06 & 34.30 & 33.99 & 34.90 & 37.10 \\
\hline N3 & 50.10 & 58.13 & 55.20 & 56.00 & 55.90 & 51.80 & 50.60 & 51.00 & 53.59 \\
\hline \multirow[t]{2}{*}{ Mean } & 36.79 & 44.36 & 40.36 & 40.92 & 41.05 & 41.13 & 36.90 & 36.09 & 39.23 \\
\hline & \multicolumn{9}{|c|}{70 DAT } \\
\hline N1 & 20.15 & 26.30 & 24.30 & 24.70 & 24.00 & 19.36 & 20.00 & 19.30 & 22.26 \\
\hline N2 & 27.40 & 33.10 & 31.50 & 32.20 & 32.60 & 27.80 & 27.90 & 26.40 & 29.86 \\
\hline N3 & 34.90 & 40.60 & 39.80 & 39.99 & 40.00 & 32.18 & 33.40 & 34.00 & 36.85 \\
\hline \multirow[t]{2}{*}{ Mean } & 27.48 & 32.35 & 31.86 & 32.29 & 32.20 & 26.44 & 27.10 & 26.56 & 29.65 \\
\hline & \multicolumn{9}{|c|}{95 DAT } \\
\hline N1 & 16.33 & 2.00 & $22 . \varepsilon$ & 22.60 & 22.05 & 17.00 & 17.35 & 17.80 & 19.74 \\
\hline N2 & 24.60 & 27.20 & 27.60 & 27.95 & 26.90 & 23.20 & 23.36 & 23.00 & 25.47 \\
\hline N3 & 28.45 & 33.00 & 33.15 & 33.70 & 32.70 & 27.50 & 27.60 & 26.90 & 30.37 \\
\hline Mean & 23.12 & 27.53 & 27.85 & 28.08 & 27.21 & 22.60 & 22.77 & 22.56 & 25.19 \\
\hline
\end{tabular}

Data indicate that in the first season at 30,50,70 and 95 DAT available $\mathrm{NH}_{4}-\mathrm{N}$ tended to increase as $\mathrm{N}$ levels increased. Plots which received high level of $\mathrm{N}$ fertilizer $\left(92 \mathrm{~kg} \mathrm{~N}^{-d^{-1}}\right)$ showed more available $\mathrm{NH}_{4}$ $\mathrm{N}$ than plots, which received low $\mathrm{N}$ levels. Data show also that the highest values of available $\mathrm{NH}_{4}^{+}-\mathrm{N}$ were obtained at 30 DAT with the application of $92 \mathrm{~kg} \mathrm{~N} \mathrm{fed}^{-1}$ while, the lowest values of $\mathrm{NH}_{4}-\mathrm{N}$ were obtained at 95 DAT with untreated soil. This view could be in harmony with those found by De Datta (1981), Suresh et al. (1995) and Sikdar et al. (2008) who stated that increasing level of nitrogen application up to $73 \mathrm{~kg} \mathrm{~N}$ fed $^{-1}$ increased the availability of $\mathrm{NH}_{4}^{+}$in soil. The same trend was found in the second season.

Regarding the affect of potassium application methods, data show that in the first season at different periods of sampling application of potassium fertilizer at transplanting increased available $\mathrm{NH}_{4}-\mathrm{N}$ compared with treatments having no potassium application at transplanting. This mainly due to the convergence radius of both the $\mathrm{NH}_{4}^{+}, \mathrm{K}^{+}$and radius of six fold gap for minerals of clay 2:1 which would lead to fixation of potassium on exchangeable sites releasing the $\mathrm{NH}_{4}$ to the soil solution. The same trend was found in the second season.

It is worthy to note that the highest values of available $\mathrm{NH}_{4}-\mathrm{N}$ in the soil were recorded at 30 DAT under $92 \mathrm{~kg} \mathrm{~N}$ fed ${ }^{-1}$ with application full dose of potassium before transplanting ( $50 \mathrm{~kg}$ potassium sulphate), while the lowest values of soil $\mathrm{NH}_{4}-\mathrm{N}$ available were observed in plots which did not receive any fertilizer at 95 DAT. 


\section{El-Hamdi, Kh. H. et al.}

\section{2- Available potassium in soil:}

Available soil potassium throughout the growth of rice plants as affected by nitrogen levels and methods of potassium application in Tables 4 and 5 .

Table 4: Available potassium in soil $\left(\mathrm{mg} \mathrm{kg}^{-1}\right)$ as affected by nitrogen levels and potassium application during 2007 season.

\begin{tabular}{|c|c|c|c|c|c|c|c|c|c|}
\hline \multirow{2}{*}{ N- Treatments } & \multicolumn{10}{|c|}{ K- Treatments } \\
\cline { 2 - 11 } & K1 & K2 & K3 & K4 & K5 & K6 & K7 & K8 & Mean \\
\hline N1 & 446 & 500 & 489 & 485 & 492 & 439 & 440 & 450 & 468 \\
\hline N2 & 490 & 570 & 550 & 543 & 555 & 480 & 495 & 499 & 523 \\
\hline N3 & 553 & 636 & 610 & 620 & 625 & 560 & 548 & 556 & 588 \\
\hline Mean & 496 & 569 & 550 & 549 & 557 & 493 & 494 & 501 & 505.3 \\
\hline \multicolumn{10}{|c|}{ 50 DAT } \\
\hline N1 & 415 & 470 & 465 & 460 & 468 & 410 & 416 & 420 & 440 \\
\hline N2 & 450 & 540 & 536 & 520 & 538 & 469 & 460 & 480 & 499 \\
\hline N3 & 530 & 600 & 584 & 570 & 599 & 535 & 528 & 540 & 599 \\
\hline Mean & 465 & 536 & 528 & 516 & 535 & 471 & 468 & 480 & 512 \\
\hline \multicolumn{10}{|c|}{70 DAT } \\
\hline N1 & 350 & 400 & 390 & 408 & 412 & 360 & 375 & 383 & 385 \\
\hline N2 & 402 & 460 & 450 & 445 & 470 & 415 & 410 & 425 & 435 \\
\hline N3 & 460 & 503 & 485 & 479 & 500 & 465 & 461 & 470 & 479 \\
\hline Mean & 404 & 454.3 & 441 & 444 & 460 & 413 & 415 & 426 & 432.2 \\
\hline \multicolumn{10}{|c|}{95 DAT } \\
\hline N1 & 300 & 325 & 310 & 312 & 305 & 295 & 305 & 300 & 306 \\
\hline N2 & 320 & 390 & 380 & 378 & 389 & 330 & 328 & 337 & 356 \\
\hline N3 & 370 & 409 & 395 & 389 & 400 & 380 & 383 & 375 & 387 \\
\hline Mean & 330 & 373 & 361 & 360 & 364 & 335 & 338 & 337 & 349 \\
\hline
\end{tabular}

Data in Tables 4 and 5 show that the highest amount of available $\mathrm{K}$ were obtained at 30 DAT in 2007 and 2008 seasons, then decreased continuously with crop growth, reaching the lowest values at 95 DAT (harvest time) in 2007 and 2008 seasons. The decrease in the available soil $\mathrm{K}$ under continuous flooding mainly attributed to that $\mathrm{K}$ uptake by rice plant and losses by leaching. Hammad (1995) stated that the availability of K decreased with continuous flooding and development of plant growth.

Data in Tables 4 and 5 show the effect of $N$ levels on available $K$ in soil was clear at early growth stage. Since in both seasons, at 30 DAT available K tended to increase with $\mathrm{N}$ level increasing. The highest mean values were obtained with application of $92 \mathrm{~kg} \mathrm{~N} \mathrm{fed}^{-1}$, while the lowest mean values were obtained with control in 2007 and 2008 seasons, after 30 DAT. Available K as affected by $\mathrm{N}$ levels have unclear trend up to $95 \mathrm{DAT}$. Wihardjaka et al. (1999) proposed that mobilization of non-exchangeable $\mathrm{K}$ in flooded rice root induced through acidification, coupled with $\mathrm{K}$ removed from the soil solution by the roots, under $\mathrm{NH}_{4}-\mathrm{N}$ nutrition, interlayer $\mathrm{K}$ can also be replaced by $\mathrm{NH}_{4}{ }^{+}$ ions which are similar in ionic size. Tisdale et al. (1985) reported that $\mathrm{NH}_{4}^{+}$ could be fixed by clays in a manner similar to that of $\mathrm{K}^{+}$. Slaton et al. (2004) stated that soil water $\mathrm{K}$ concentrations peak about on two week after flooding. 
Decline rapidly until 4 to 5 weeks after flooding and then reach a consistently low concentration for the duration of the seasons. Both soil $\mathrm{K}$ pools, exchangeable and solution reach low $\mathrm{K}$ concentration near the time of panicle differentiation and persist until the water is drained for harvest.

Data also show that in the first season at all growth periods, application of potassium before transplanting increased available $\mathrm{K}^{+}$compared with treatments having no potassium application before transplanting. Data show also that the maximum mean values of available $\mathrm{K}$ were obtained at 30 DAT with application of potassium full dose at transplanting $(50 \mathrm{~kg}$ potassium sulphate), while the lowest values of available $\mathrm{K}$ were observed at 95 DAT with untreated control. Thippeswamy et al. (2000) reported that water soluble $\mathrm{K}$ and available $\mathrm{K}^{+}$increased with increase in potassium doses up to $80 \mathrm{~kg}$ $\mathrm{K}_{2} \mathrm{O} \mathrm{ha}^{-1}$, but decreased with growth stages of crop from tillering to harvesting. The same trend was found in the second seasons.

Concerning the interaction effect, data in Tables 4 and 5 , show that highest values of soil $\mathrm{K}$ available in soil $\left(636\right.$ and $\left.660 \mathrm{mg} \mathrm{kg}^{-1}\right)$ were obtained at 30 DAT when rice plants were fertilized with $92 \mathrm{~kg} \mathrm{~N} \mathrm{fed}^{-1}$ and application full dose of potassium before transplanting, in 2007 and 2008 seasons, respectively. While the lowest values of soil $\mathrm{K}$ available (300 and $299 \mathrm{mg} \mathrm{kg}^{-1}$ in 2007 and 2008, respectively) were observed at (95 DAT) in both seasons of study.

Table 5: Available potassium in soil $\left(\mathrm{mg} \mathrm{kg}^{-1}\right)$ as affected by nitrogen levels and potassium application during 2008 seasons.

\begin{tabular}{|c|c|c|c|c|c|c|c|c|c|}
\hline \multirow{10}{*}{ N- Treatments } & \multicolumn{10}{|c|}{ K- Treatments } \\
\cline { 2 - 11 } & K1 & K2 & K3 & K4 & K5 & K6 & K7 & K8 & Mean \\
\hline N1 & 459 & 515 & 496 & 499 & 505 & 445 & 452 & 458 & 478 \\
\hline N2 & 520 & 595 & 568 & 560 & 566 & 518 & 510 & 522 & 545 \\
\hline N3 & 580 & 660 & 626 & 620 & 630 & 583 & 585 & 577 & 608 \\
\hline Mean & 520 & 590 & 563 & 559 & 567 & 515 & 516 & 519 & 543 \\
\hline \multicolumn{10}{|c|}{ 50 DAT } \\
\hline N1 & 420 & 478 & 463 & 462 & 466 & 415 & 413 & 418 & 442 \\
\hline N2 & 452 & 546 & 538 & 523 & 537 & 463 & 459 & 470 & 498 \\
\hline N3 & 536 & 609 & 590 & 578 & 592 & 536 & 530 & 535 & 563 \\
\hline Mean & 469 & 544 & 530 & 521 & 532 & 471 & 476 & 474 & 501 \\
\hline \multicolumn{10}{|c|}{70 DAT } \\
\hline N1 & 351 & 405 & 393 & 395 & 414 & 363 & 366 & 370 & 333 \\
\hline N2 & 406 & 465 & 451 & 450 & 469 & 417 & 415 & 420 & 386 \\
\hline N3 & 463 & 519 & 490 & 483 & 511 & 466 & 460 & 469 & 482 \\
\hline Mean & 407 & 463 & 445 & 442 & 465 & 415 & 414 & 419 & 434.13 \\
\hline \multicolumn{10}{|c|}{95 DAT } \\
\hline N1 & 299 & 326 & 319 & 323 & 325 & 299 & 300 & 302 & 312 \\
\hline N2 & 329 & 395 & 385 & 382 & 396 & 331 & 330 & 334 & 360 \\
\hline N3 & 374 & 409 & 399 & 392 & 410 & 381 & 380 & 379 & 390 \\
\hline Mean & 336 & 376 & 368 & 366 & 377 & 337 & 336 & 338 & 354 \\
\hline
\end{tabular}




\section{El-Hamdi, Kh. H. et al.}

\section{2- Effect of nitrogen and potassium fertilization on nutrients uptake:}

Nitrogen uptake in hybrid rice at different growth periods as affected by different nitrogen levels and methods of potassium application during 2007 and 2008 seasons.

The results in Tables 6 and 7 show that increase $\mathrm{N}$ levels up to $92 \mathrm{~kg} \mathrm{~N}$ fed $^{-1}$ increased $\mathrm{N}$ uptake at all studied growth periods in 2007 and 2008 seasons. It might be owing to fact that hybrid rice absorbs $\mathrm{N}$ continuously up to maturity and the delayed $\mathrm{N}$ application at flowering stage expectedly results in relatively higher $\mathrm{N}$ accumulation in foliage including lower leaves. This probably contributing to higher growth leading to lager cytokinine production which causing more dry matter production to adequately meet the needs arising on account of larger sink in hybrids this undoubtedly would increase the uptake of nitrogen by plant, (Krishankumar et al. 2005). These results are in harmony with those obtained by Meena et al. (2002) and Zhang Hong et al. (2003).

Addition of $\mathrm{K}$ as full dose before transplanting $(50 \mathrm{~kg}$ potassium sulphate) increased significantly nitrogen uptake at 30 days after transplanting (DAT) in both seasons, Table 6 and 7.

Data also show that application of potassium (half dose before transplanting (25 kg potassium sulphate) + spray with $2 \% \quad \mathrm{~K}_{2} \mathrm{O}(4 \mathrm{~kg}$ potassium sulphate) at panicle initiation stage + spray with $2 \% \mathrm{~K} 2 \mathrm{O}(4 \mathrm{~kg}$ potassium sulphate) at flowering stages) increased significantly the nitrogen uptake at 30,50 and 95 DAT in both seasons. This might be to the continuous supply of $\mathrm{K}$ to the crop during growth period which is more beneficial and increased dry matter accumulation and fertilizer use efficiency resulted higher $\mathrm{N}$ uptake. These findings are in close conformity with those of Thakur et al. (1999).

Table 6: Nitrogen uptake $\left(\mathrm{Kg} \mathrm{fed}^{-1}\right)$ at different growth periods $(30,50$, 70,95 DAT) as affected by different nitrogen levels and methods of potassium application during 2007 season.

\begin{tabular}{|c|c|c|c|c|c|}
\hline \multirow{2}{*}{ Treatment } & \multirow{2}{*}{30 DAT } & \multirow{2}{*}{50 DAT } & \multirow{2}{*}{70 DAT } & \multicolumn{2}{|c|}{95 DAT } \\
\hline & & & & Grain & Straw \\
\hline \multicolumn{6}{|l|}{$\mathrm{N}$ - treatments } \\
\hline$N_{1}$ & $21.91 \mathrm{c}$ & $41.45 \mathrm{c}$ & $56.49 \mathrm{c}$ & $34.37 \mathrm{c}$ & $24.88 \mathrm{c}$ \\
\hline $\mathrm{N}_{2}$ & $53.84 \mathrm{~b}$ & $79.85 b$ & $100.45 b$ & $54.07 \mathrm{~b}$ & $33.68 \mathrm{~b}$ \\
\hline $\mathbf{N}_{3}$ & $77.61 \mathrm{a}$ & $113.05 \mathrm{a}$ & $123.64 \mathrm{a}$ & $63.82 \mathrm{a}$ & $43.0 \mathrm{a}$ \\
\hline \multicolumn{6}{|l|}{$\mathrm{K}$ - treatments } \\
\hline $\mathrm{K}_{1}$ & $43.12 \mathrm{c}$ & $64.37 \mathrm{c}$ & $80.91 \mathrm{~h}$ & $43.39 \mathrm{e}$ & $30.05 c$ \\
\hline $\mathrm{K}_{2}$ & $62.11 \mathrm{a}$ & $88.21 \mathrm{a}$ & $103.68 \mathrm{~b}$ & $55.61 \mathrm{~b}$ & $35.16 \mathrm{~b}$ \\
\hline $\mathrm{K}_{3}$ & $57.63 \mathrm{~b}$ & $88.30 \mathrm{a}$ & $98.22 \mathrm{c}$ & $54.12 \mathrm{c}$ & $34.86 \mathrm{ab}$ \\
\hline $\mathrm{K}_{4}$ & $57.81 \mathrm{~b}$ & $77.44 \mathrm{~b}$ & $93.22 \mathrm{~d}$ & $53.06 \mathrm{c}$ & $33.99 \mathrm{~b}$ \\
\hline $\mathrm{K}_{5}$ & $58.08 \mathrm{~b}$ & $89.33 \mathrm{a}$ & $106.00 \mathrm{a}$ & $59.12 \mathrm{a}$ & $36.03 a$ \\
\hline $\mathrm{K}_{6}$ & $43.72 \mathrm{c}$ & $76.52 \mathrm{~b}$ & $89 . .49 \mathrm{f}$ & $46.88 \mathrm{~d}$ & $33.55 b$ \\
\hline$K_{7}$ & $43.12 \mathrm{c}$ & $64.51 \mathrm{c}$ & $84.65 \mathrm{~g}$ & $46.17 \mathrm{~d}$ & $33.43 \mathrm{~b}$ \\
\hline $\mathrm{K}_{8}$ & $43.03 \mathrm{c}$ & $76.28 \mathrm{~b}$ & $91.83 \mathrm{e}$ & $47.67 \mathrm{~d}$ & $33.76 b$ \\
\hline
\end{tabular}


Table 7: Nitrogen uptake $\left(\mathrm{Kg} \mathrm{fed}^{-1}\right)$ at different growth periods as affected by different nitrogen levels and methods of potassium application during 2008 season.

\begin{tabular}{|c|c|c|c|c|c|}
\hline \multirow{2}{*}{ Treatment } & \multirow{2}{*}{30 DAT } & \multirow{2}{*}{50 DAT } & \multirow{2}{*}{ 70 DAT } & \multicolumn{2}{|c|}{ 95 DAT } \\
\cline { 5 - 6 } & & & & Grain & Straw \\
\hline N- treatments & & & & & \\
\hline $\mathbf{N}_{\mathbf{1}}$ & $22.44 \mathrm{c}$ & $41.23 \mathrm{c}$ & $58.05 \mathrm{c}$ & $35.44 \mathrm{c}$ & $25.25 \mathrm{c}$ \\
\hline $\mathbf{N}_{\mathbf{2}}$ & $54.74 \mathrm{~b}$ & $80.85 \mathrm{~b}$ & $102.62 \mathrm{~b}$ & $56.00 \mathrm{~b}$ & $35.16 \mathrm{~b}$ \\
\hline $\mathbf{N}_{\mathbf{3}}$ & $78.82 \mathrm{a}$ & $115.30 \mathrm{a}$ & $125.26 \mathrm{a}$ & $65.33 \mathrm{a}$ & $43.72 \mathrm{a}$ \\
\hline K- treatments & & & & & \\
\hline $\mathbf{K}_{\mathbf{1}}$ & $44.58 \mathrm{~d}$ & $66.01 \mathrm{~d}$ & $82.63 \mathrm{~g}$ & $44.86 \mathrm{e}$ & $31.00 \mathrm{~d}$ \\
\hline $\mathbf{K}_{\mathbf{2}}$ & $62.78 \mathrm{a}$ & $89.67 \mathrm{a}$ & $104.59 \mathrm{~b}$ & $57.50 \mathrm{~b}$ & $36.58 \mathrm{ab}$ \\
\hline $\mathbf{K}_{\mathbf{3}}$ & $59.40 \mathrm{~b}$ & $88.58 \mathrm{a}$ & $100.06 \mathrm{c}$ & $55.60 \mathrm{c}$ & $35.22 \mathrm{bc}$ \\
\hline $\mathbf{K}_{\mathbf{4}}$ & $57.58 \mathrm{c}$ & $79.22 \mathrm{~b}$ & $94.68 \mathrm{~d}$ & $55.01 \mathrm{c}$ & $34.32 \mathrm{c}$ \\
\hline $\mathbf{K}_{\mathbf{5}}$ & $59.35 \mathrm{~b}$ & $88.65 \mathrm{a}$ & $108.26 \mathrm{a}$ & $60.57 \mathrm{a}$ & $37.76 \mathrm{a}$ \\
\hline $\mathbf{K}_{\mathbf{6}}$ & $44.93 \mathrm{~d}$ & $76.20 \mathrm{c}$ & $91.43 \mathrm{e}$ & $48.44 \mathrm{~d}$ & $34.93 \mathrm{bc}$ \\
\hline $\mathbf{K}_{\mathbf{7}}$ & $43.43 \mathrm{~d}$ & $66.56 \mathrm{~d}$ & $87.0 \mathrm{f}$ & $47.35 \mathrm{~d}$ & $33.66 \mathrm{c}$ \\
\hline $\mathbf{K}_{\mathbf{8}}$ & $43.96 \mathrm{~d}$ & $78.11 \mathrm{bc}$ & $82.63 \mathrm{~g}$ & $48.75 \mathrm{~d}$ & $34.21 \mathrm{c}$ \\
\hline
\end{tabular}

Data listed in Tables 8 and 9 show that highly significantly interaction differences were recorded between nitrogen levels and methods of potassium application at growth periods in both seasons of study. The highest values of nitrogen uptake (90 and $91 \mathrm{~kg} \mathrm{~N} . \mathrm{fed}-1$ ) were recorded when rice plants were fertilized with $92 \mathrm{~kg} \mathrm{~N} \mathrm{fed}^{-1}$ in combined with application of potassium full dose before transplanting $\left(\mathrm{K}_{2}\right)$ at 30 DAT in 2007 and 2008 seasons, respectively . Data show also that maximum nitrogen uptake values were found when $92 \mathrm{~kg}$ $\mathrm{N}$ fed $^{-1}$ was combined with application of potassium (half dose before transplanting (25 kg potassium sulphate) + spray with $2 \%(4 \mathrm{~kg}$ potassium sulphate) at panicle initiation stage + spray with $2 \%(4 \mathrm{~kg}$ potassium sulphate) at flowering stages), which increase significantly the $\mathrm{N}$ uptake at 50, 70 and 95 DAT in both seasons of study. This might be owing to the continuous supply of potassium to the crop growth period which is more beneficial and increase nitrogen transport amount and percentage after anthesis, as well as, nitrogen accumulation in different rice organs. (Wang et al.2004). These results are in congruent with that the increase of nitrogen uptake could be attributed to the role of nitrogen and potassium metabolism in rice plant. 
El-Hamdi, Kh. H. et al.

Table 8: Nitrogen uptake $\left(\mathrm{Kg} \mathrm{fed}^{-1}\right)$ at different growth periods $(30,50,70$ and 95 DAT) as affected by interaction between different nitrogen levels and methods of potassium application during 2007 and 2008 seasons.

\begin{tabular}{|c|c|c|c|c|c|c|c|}
\hline \multirow{3}{*}{\multicolumn{2}{|c|}{ K-treatments }} & \multicolumn{6}{|c|}{$\mathrm{N}$ - treatments } \\
\hline & & \multicolumn{3}{|c|}{2007} & \multicolumn{3}{|c|}{2008} \\
\hline & & $N_{1}$ & $\mathrm{~N}_{2}$ & $\mathbf{N}_{3}$ & $N_{1}$ & $\mathbf{N}_{2}$ & $\mathbf{N}_{3}$ \\
\hline \multirow{8}{*}{$\begin{array}{l}\text { 匙 } \\
\text { 足 }\end{array}$} & $K_{1}$ & $15.98 \mathrm{~g}$ & $45.24 \mathrm{e}$ & $68.14 c$ & $17.51 \mathrm{j}$ & $46.34 \mathrm{~g}$ & $69.89 \mathrm{~cd}$ \\
\hline & $K_{2}$ & $30.51 f$ & $65.83 \mathrm{~cd}$ & $90.00 \mathrm{a}$ & $31.07 \mathrm{~h}$ & $66.26 \mathrm{e}$ & $91.02 \mathrm{a}$ \\
\hline & $\mathrm{K}_{3}$ & $27.21 f$ & $61.52 d$ & $84.46 \mathrm{~b}$ & $27.00 \mathrm{i}$ & $65.46 \mathrm{e}$ & $85.75 b$ \\
\hline & $\mathrm{K}_{4}$ & $26.49 f$ & $62.09 d$ & $84.86 \mathrm{~b}$ & $26.60 \mathrm{i}$ & $59.59 f$ & $86.48 b$ \\
\hline & $\mathrm{K}_{5}$ & $26.97 f$ & $62.17 d$ & $84.96 \mathrm{~b}$ & $27.15 \mathrm{i}$ & $65.34 \mathrm{e}$ & $85.56 \mathrm{~b}$ \\
\hline & $\mathrm{K}_{6}$ & $15.97 \mathrm{~g}$ & $45.40 \mathrm{e}$ & $69.80 \mathrm{c}$ & $16.64 \mathrm{j}$ & $45.71 \mathrm{~g}$ & $72.43 c$ \\
\hline & $K_{7}$ & $16.39 \mathrm{~g}$ & $44.48 \mathrm{e}$ & $69.39 c$ & $16.56 \mathrm{j}$ & $43.67 \mathrm{~g}$ & $70.06 \mathrm{~cd}$ \\
\hline & $K_{8}$ & $15.79 \mathrm{~g}$ & $44.02 \mathrm{e}$ & $69.27 \mathrm{c}$ & $16.90 \mathrm{j}$ & $45.59 \mathrm{~g}$ & $69.39 d$ \\
\hline & \multirow{3}{*}{ K-treatments } & \multicolumn{6}{|c|}{$\mathrm{N}$ - Treatments } \\
\hline & & & 2007 & & & 2008 & \\
\hline & & $N_{1}$ & $\mathbf{N}_{2}$ & $\mathbf{N}_{3}$ & $N_{1}$ & $\mathbf{N}_{2}$ & $\mathrm{~N}_{3}$ \\
\hline \multirow{8}{*}{$\begin{array}{l}\text { 䢍 } \\
\text { ட }\end{array}$} & $\mathrm{K}_{1}$ & $33.56 \mathrm{j}$ & $62.08 \mathrm{e}$ & $97.46 \mathrm{c}$ & $32.76 \mathrm{k}$ & $64.77 \mathrm{~g}$ & $100.50 d$ \\
\hline & $\mathrm{K}_{2}$ & $47.56 f$ & $92.20 \mathrm{a}$ & $124.87 a$ & $46.30 \mathrm{~h}$ & $95.74 \mathrm{e}$ & $126.76 \mathrm{a}$ \\
\hline & $\mathrm{K}_{3}$ & $46.28 \mathrm{fg}$ & $93.26 a$ & $125.36 a$ & $45.16 \mathrm{hi}$ & $93.30 \mathrm{e}$ & $127.30 \mathrm{a}$ \\
\hline & $\mathrm{K}_{4}$ & $43.54 \mathrm{fg}$ & $75.36 \mathrm{~b}$ & $113.42 \mathrm{~b}$ & $42.53 i j$ & $79.30 \mathrm{f}$ & $115.83 \mathrm{~b}$ \\
\hline & $K_{5}$ & 45.33fgh & $97.49 a$ & $125.17 \mathrm{a}$ & 45.56hi & $93.60 \mathrm{e}$ & $126.80 \mathrm{a}$ \\
\hline & $\mathrm{K}_{6}$ & $41.25 \mathrm{gh}$ & $78.65 \mathrm{~b}$ & $109.66 b$ & $40.30 \mathrm{j}$ & $77.00 f$ & $111.30 \mathrm{c}$ \\
\hline & $\mathrm{K}_{7}$ & $33.82 i$ & $62.53 \mathrm{c}$ & $97.19 \mathrm{c}$ & $34.50 \mathrm{k}$ & $65.00 \mathrm{~g}$ & $100.20 d$ \\
\hline & $\mathrm{K}_{8}$ & $40.26 \mathrm{~h}$ & $77.29 d$ & $111.28 b$ & $42.74 h-j$ & $78.10 \mathrm{f}$ & $113.50 \mathrm{bc}$ \\
\hline \multirow{3}{*}{\multicolumn{2}{|c|}{ K-treatments }} & \multicolumn{6}{|c|}{$\mathrm{N}$ - treatments } \\
\hline & & & 2007 & & & 2008 & \\
\hline & & $N_{1}$ & $\mathbf{N}_{2}$ & $\mathbf{N}_{3}$ & $N_{1}$ & $\mathbf{N}_{2}$ & $\mathbf{N}_{3}$ \\
\hline & $K_{1}$ & $50.05 p$ & $82.06 \mathrm{~L}$ & $110.63 \mathrm{gh}$ & $51.20 n$ & $83.10 \mathrm{j}$ & 113.60ef \\
\hline \multirow{7}{*}{$\begin{array}{l}\text { 芯 } \\
\text { D } \\
\text { R }\end{array}$} & $\mathrm{K}_{2}$ & $62.43 \mathrm{~m}$ & $112.66 \mathrm{fg}$ & $135.96 b$ & $64.15 \mathrm{k}$ & $114.83 \mathrm{e}$ & $134.80 \mathrm{~b}$ \\
\hline & $\mathrm{K}_{3}$ & $60.22 \mathrm{~m}$ & $109.0 \mathrm{~h}$ & $126.00 c$ & $61.22 \mathrm{kl}$ & $110.96 f$ & $128.00 \mathrm{c}$ \\
\hline & $\mathrm{K}_{4}$ & $58.00 n$ & $96.73 j$ & $124.16 \mathrm{c}$ & $58.25 \mathrm{Im}$ & $98.60 \mathrm{~h}$ & $127.20 \mathrm{c}$ \\
\hline & $\mathrm{K}_{5}$ & $62.21 \mathrm{~m}$ & 113.6ef & $142.16 \mathrm{a}$ & $64.90 \mathrm{k}$ & $115.60 \mathrm{e}$ & $144.20 \mathrm{a}$ \\
\hline & $\mathrm{K}_{6}$ & 54.300 & $98.65 j$ & $115.53 \mathrm{e}$ & $55.90 \mathrm{~m}$ & $100.9 \mathrm{gh}$ & $117.50 \mathrm{de}$ \\
\hline & $\mathrm{K}_{7}$ & $50.51 p$ & $88.53 \mathrm{k}$ & $114.93 \mathrm{ef}$ & $51.50 n$ & $92.50 \mathrm{i}$ & $117.00 \mathrm{de}$ \\
\hline & $\mathrm{K}_{8}$ & $50.05 p$ & $102.28 \mathrm{i}$ & $118.96 d$ & $57.30 \mathrm{~m}$ & $104.40 \mathrm{~g}$ & $119.80 d$ \\
\hline
\end{tabular}


Table 9: Nitrogen uptake $\left(\mathrm{Kg} \mathrm{fed}^{-1}\right)$ at 95 DAT (grain and straw) as affected by interaction between different nitrogen levels and methods of potassium application during 2007 and 2008 seasons.

\begin{tabular}{|c|c|c|c|c|c|c|c|}
\hline \multirow{11}{*}{ 高 } & \multirow{2}{*}{ K-treatments } & \multicolumn{6}{|c|}{$\mathrm{N}$ - treatments } \\
\hline & & \multicolumn{3}{|c|}{2007} & \multicolumn{3}{|c|}{2008} \\
\hline & & $\mathbf{N}_{1}$ & $\mathbf{N}_{2}$ & $\mathbf{N}_{3}$ & $\mathbf{N}_{1}$ & $\mathbf{N}_{2}$ & $\mathbf{N}_{3}$ \\
\hline & $\mathrm{K}_{1}$ & $26.28 \mathrm{k}$ & $45.00 \mathrm{~g}$ & $58.90 \mathrm{de}$ & $27.60 n$ & $48.00 \mathrm{j}$ & $59.00 \mathrm{~g}$ \\
\hline & $\mathrm{K}_{2}$ & $40.68 \mathrm{~h}$ & $59.36 \mathrm{de}$ & $66.80 \mathrm{~b}$ & $41.20 \mathrm{k}$ & $61.00 \mathrm{efg}$ & $70.10 \mathrm{~b}$ \\
\hline & $\mathrm{K}_{3}$ & $38.28 \mathrm{i}$ & 58.8de & $65.30 \mathrm{~b}$ & 39.501 & $60.00 \mathrm{fg}$ & $67.30 \mathrm{c}$ \\
\hline & $\mathrm{K}_{4}$ & $37.60 \mathrm{i}$ & $57.5 \mathrm{e}$ & $64.10 \mathrm{bc}$ & $38.85 \mid$ & $60.40 \mathrm{fg}$ & $65.80 \mathrm{~cd}$ \\
\hline & $\mathrm{K}_{5}$ & $41.66 \mathrm{~h}$ & $61.90 \mathrm{~cd}$ & $73.80 a$ & $42.90 \mathrm{k}$ & $65.00 \mathrm{~d}$ & $73.81 a$ \\
\hline & $\mathrm{K}_{6}$ & $30.50 \mathrm{j}$ & $50.16 f$ & 60.00de & $31.72 \mathrm{~m}$ & 51.60hi & $62.00 \mathrm{ef}$ \\
\hline & $\mathrm{K}_{7}$ & $29.18 j$ & $49.00 f$ & $60.35 \mathrm{de}$ & $30.36 \mathrm{~m}$ & $50.00 i$ & $61.70 \mathrm{ef}$ \\
\hline & $\mathrm{K}_{8}$ & $30.80 \mathrm{j}$ & $50.90 f$ & $61.33 d$ & $31.25 \mathrm{~m}$ & $52.00 \mathrm{~h}$ & $63.00 \mathrm{e}$ \\
\hline \multirow{11}{*}{ 离 } & \multirow{3}{*}{ K-treatments } & \multicolumn{6}{|c|}{$\mathrm{N}$ - treatments } \\
\hline & & \multicolumn{3}{|c|}{2007} & \multicolumn{3}{|c|}{2008} \\
\hline & & $\mathbf{N}_{1}$ & $\mathbf{N}_{2}$ & $\mathbf{N}_{3}$ & $\mathbf{N}_{1}$ & $\mathbf{N}_{\mathbf{2}}$ & $\mathbf{N}_{3}$ \\
\hline & $\mathrm{K}_{1}$ & $20.50 \mathrm{~h}$ & $30.66 \mathrm{e}$ & $39.00 c$ & $21.00 \mathrm{j}$ & $32.00 \mathrm{~g}$ & $40.00 \mathrm{~cd}$ \\
\hline & $\mathrm{K}_{2}$ & $26.60 \mathrm{fg}$ & $34.80 \mathrm{~d}$ & $44.10 a b$ & $27.90 \mathrm{~h}$ & $36.25 \mathrm{ef}$ & $45.60 \mathrm{ab}$ \\
\hline & $\mathrm{K}_{3}$ & $26.45 \mathrm{fg}$ & $34.35 d$ & 43.80ab & 26.00hi & $34.66 \mathrm{fg}$ & 45.00ab \\
\hline & $\mathrm{K}_{4}$ & $24.98 f g$ & $34.00 \mathrm{~d}$ & 43.00ab & 23.66ij & $35.50 \mathrm{efg}$ & 43.80ab \\
\hline & $\mathrm{K}_{5}$ & $27.50 f$ & $35.00 \mathrm{~d}$ & $45.60 \mathrm{a}$ & 28.00h & 38.50de & $46.80 \mathrm{a}$ \\
\hline & $\mathrm{K}_{6}$ & $24.55 \mathrm{~g}$ & $33.50 \mathrm{~d}$ & $42.60 \mathrm{~b}$ & 25.80hi & 36.00ef & $43.00 \mathrm{bc}$ \\
\hline & $\mathrm{K}_{7}$ & $24.40 \mathrm{~g}$ & 33.00de & $42.90 \mathrm{ab}$ & 25.00hi & $34.00 \mathrm{fg}$ & $42.00 \mathrm{bc}$ \\
\hline & $\mathrm{K}_{8}$ & $24.09 \mathrm{~g}$ & $34.20 d$ & 43.00ab & 24.66hi & $34.36 \mathrm{fg}$ & 43.60ab \\
\hline
\end{tabular}

\subsection{Potassium uptake:}

Potassium uptake $\left(\mathrm{kg} \mathrm{fed}^{-1}\right)$ by hybrid rice at different growth periods as affected by different nitrogen levels and methods of potassium application during 2007 and 2008 are presented in Tables 10,11,12 and 13.

Data in tables 10 and 11 reveal that potassium uptake by hybrid rice in both years of study, nitrogen fertilization significantly increased potassium uptake at all growth periods (30, 50, 70 and 95 DAT). Each successive increment of nitrogen resulted in a significant increase in potassium uptake over the preceding level, the highest potassium uptake was recorded at $92 \mathrm{~kg}$ $\mathrm{N}$ fed ${ }^{-1}$. These increases in potassium uptake by hybrid rice plants may be due to the role of nitrogen in improving nutrients absorption. These results are in agreement with those of Meena et al. (2002), Maiti et al. (2003) and Dwivedi et al (2006).

Data in Tables 10 and 11 indicate that potassium uptake was significant affect by a method of potassium application which observed at 30 DAT by applying full dose of potassium before transplanting. On the other hand low potassium uptake was found in plots which did not receive potassium fertilizer at transplanting. As regarded application of potassium half dose at transplanting (25 kg potassium sulphate) + spray with $2 \%(4 \mathrm{~kg}$ potassium sulphate) at panicle initiation stage + spray with $2 \%(4 \mathrm{~kg}$ potassium sulphate) at flowering stages increased significantly the potassium uptake at 50, 70 and 95 DAT in both seasons. 
El-Hamdi, Kh. H. et al.

Table 10: Potassium uptake $\left(\mathrm{Kg} \mathrm{fed}^{-1}\right)$ at different growth periods as affected by different nitrogen levels and methods of potassium application during 2007 season.

\begin{tabular}{|c|c|c|c|c|c|}
\hline \multirow{2}{*}{ Treatment } & \multirow{2}{*}{30 DAT } & \multirow{2}{*}{50 DAT } & \multirow{2}{*}{70 DAT } & \multicolumn{2}{|c|}{95 DAT } \\
\hline & & & & Grain & Straw \\
\hline \multicolumn{6}{|l|}{$\mathrm{N}$ treatments } \\
\hline $\mathrm{N}_{1}$ & $29.26 c$ & $57.57 \mathrm{c}$ & $86.63 \mathrm{c}$ & $12.82 \mathrm{c}$ & $74.89 \mathrm{c}$ \\
\hline $\mathbf{N}_{2}$ & $57.13 b$ & $84.79 b$ & $121.34 b$ & $19.06 \mathrm{~b}$ & $102.10 \mathrm{~b}$ \\
\hline $\mathbf{N}_{3}$ & $87.48 a$ & $119.24 a$ & $158.10 a$ & $23.47 a$ & $112.56 a$ \\
\hline \multicolumn{6}{|l|}{$\mathrm{K}$ treatments } \\
\hline $\mathrm{K}_{1}$ & $45.93 c$ & $68.61 d$ & $102.10 \mathrm{~g}$ & $14.04 \mathrm{e}$ & $85.83 f$ \\
\hline $\mathrm{K}_{2}$ & $73.97 a$ & $101.83 a$ & $135.00 \mathrm{~b}$ & $20.34 a b$ & $101.05 b$ \\
\hline $\mathrm{K}_{3}$ & $68.32 b$ & $101.17 a$ & $129.33 c$ & $19.21 b c$ & $99.33 c$ \\
\hline $\mathrm{K}_{4}$ & $69.10 \mathrm{~b}$ & $91.16 \mathrm{~b}$ & $124.61 d$ & $18.72 \mathrm{bc}$ & $96.66 \mathrm{~d}$ \\
\hline $\mathrm{K}_{5}$ & $69.20 b$ & $101.16 a$ & $139.60 a$ & $21.60 \mathrm{a}$ & $104.90 a$ \\
\hline $\mathrm{K}_{6}$ & $46.07 c$ & $81.22 \mathrm{c}$ & $114.25 \mathrm{e}$ & $18.06 \mathrm{~cd}$ & $94.40 \mathrm{e}$ \\
\hline $\mathrm{K}_{7}$ & $46.00 \mathrm{c}$ & $69.22 \mathrm{~d}$ & $108.86 f$ & $16.88 \mathrm{~d}$ & $94.13 e$ \\
\hline $\mathrm{K}_{8}$ & $45.07 c$ & $82.31 c$ & $122.46 d$ & $18.76 \mathrm{bc}$ & $95.83 d$ \\
\hline
\end{tabular}

Table 11: Potassium uptake $\left(\mathrm{Kg} \mathrm{fed}^{-1}\right)$ at different growth periods as affected by different nitrogen levels and methods of potassium application during 2008 season.

\begin{tabular}{|c|c|c|c|c|c|}
\hline \multirow{2}{*}{ Treatment } & \multirow{2}{*}{ 30 DAT } & \multirow{2}{*}{ 50 DAT } & \multirow{2}{*}{ 70 DAT } & \multicolumn{2}{|c|}{ 95 DAT } \\
\cline { 5 - 6 } & & & & Grain & Straw \\
\hline N- treatments & & & & & \\
\hline $\mathbf{N}_{\mathbf{1}}$ & $31.77 \mathrm{c}$ & $58.56 \mathrm{c}$ & $87.41 \mathrm{c}$ & $13.14 \mathrm{c}$ & $76.48 \mathrm{c}$ \\
\hline $\mathbf{N}_{\mathbf{2}}$ & $58.96 \mathrm{~b}$ & $86.73 \mathrm{~b}$ & $123.27 \mathrm{~b}$ & $19.79 \mathrm{~b}$ & $104.98 \mathrm{~b}$ \\
\hline $\mathbf{N}_{\mathbf{3}}$ & $88.55 \mathrm{a}$ & $120.04 \mathrm{a}$ & $157.27 \mathrm{a}$ & $23.49 \mathrm{a}$ & $116.63 \mathrm{a}$ \\
\hline K- treatments & & & & & \\
\hline $\mathbf{K}_{\mathbf{1}}$ & $47.43 \mathrm{c}$ & $69.26 \mathrm{e}$ & $102.74 \mathrm{~h}$ & $14.55 \mathrm{~d}$ & $87.83 \mathrm{f}$ \\
\hline $\mathbf{K}_{\mathbf{2}}$ & $75.56 \mathrm{a}$ & $102.06 \mathrm{a}$ & $136.01 \mathrm{~b}$ & $20.56 \mathrm{~b}$ & $104.67 \mathrm{~b}$ \\
\hline $\mathbf{K}_{\mathbf{3}}$ & $70.45 \mathrm{~b}$ & $101.06 \mathrm{a}$ & $129.88 \mathrm{c}$ & $19.35 \mathrm{bc}$ & $100.83 \mathrm{c}$ \\
\hline $\mathbf{K}_{\mathbf{4}}$ & $70.82 \mathrm{~b}$ & $92.80 \mathrm{~b}$ & $125.43 \mathrm{~d}$ & $19.17 \mathrm{bc}$ & $100.01 \mathrm{~cd}$ \\
\hline $\mathbf{K}_{\mathbf{5}}$ & $70.78 \mathrm{~b}$ & $103.27 \mathrm{a}$ & $140.60 \mathrm{a}$ & $22.27 \mathrm{a}$ & $107.31 \mathrm{a}$ \\
\hline $\mathbf{K}_{\mathbf{6}}$ & $47.31 \mathrm{c}$ & $82.81 \mathrm{~d}$ & $114.86 \mathrm{f}$ & $18.08 \mathrm{c}$ & $98.43 \mathrm{~d}$ \\
\hline $\mathbf{K}_{\mathbf{7}}$ & $47.75 \mathrm{c}$ & $70.48 \mathrm{e}$ & $109.36 \mathrm{~g}$ & $17.66 \mathrm{c}$ & $95.94 \mathrm{e}$ \\
\hline $\mathbf{K}_{\mathbf{8}}$ & $47.97 \mathrm{c}$ & $85.31 \mathrm{c}$ & $122.33 \mathrm{e}$ & $18.82 \mathrm{bc}$ & $99.90 \mathrm{~cd}$ \\
\hline
\end{tabular}

The increase of potassium uptake of hybrid rice owing to potassium fertilizer application at recommended rate having three times of application (half before transplanting (25 kg potassium sulphate) + spray with $2 \%(4 \mathrm{~kg}$ potassium sulphate) at panicle initiation stage + spray with $2 \%(4 \mathrm{~kg}$ potassium sulphate) at flowering stages were attributed directly to continuous supply which was proved more beneficial and increased dry matter resulted higher potassium uptake Thakur et al.(1999) and Ali et al.(2005) and Pattanayak et al.(2008).

Highly significant interaction differences between nitrogen levels and methods of potassium application in regarded to the potassium uptake $(\mathrm{kg}$ $\mathrm{fed}^{-1}$ ) by hybrid rice in both seasons as listed in Tables 12 and 13. Multiple range tests partly indicated that the best combination which produced the highest potassium uptake by hybrid rice at 30 DAT was $92 \mathrm{~kg} \mathrm{~N}^{-1}$ with 
application of potassium full dose at transplanting ( $50 \mathrm{~kg}$ potassium sulphate) in both seasons. On the other hand low potassium uptake was found in plots which did not receive nitrogen or potassium fertilizer in both seasons of study. At 50,70 and 95 DAT the best combination which produced the highest potassium uptake by hybrid rice was $92 \mathrm{~kg} \mathrm{~N}^{-1}$ with application of potassium half dose before transplanting $(25 \mathrm{~kg}$ potassium sulphate) + spray with $2 \%(4 \mathrm{~kg}$ potassium sulphate) at panicle initiation stage + spray with $2 \%$ (4 $\mathrm{kg}$ potassium sulphate) at flowering stages. Devendra et al. (1999) reported that application of nitrogen and potassium caused an increase in uptake of potassium in hybrid rice. Arivazhagan and Ravichandran (2005) found that application of potassium and nitrogen in split doses resulted in higher potassium uptake.

Table 12: Potassium uptake $\left(\mathrm{Kg} \mathrm{fed}^{-1}\right)$ at different growth periods as affected by interaction between different nitrogen levels and methods of potassium application during 2007 and 2008 seasons.

\begin{tabular}{|c|c|c|c|c|c|c|c|}
\hline \multirow{11}{*}{\begin{tabular}{|l}
$\mid$ \\
$\qquad$ \\
\\
\\
\end{tabular}} & \multirow{4}{*}{\begin{tabular}{|l} 
K-treatments \\
$\mathrm{K}_{1}$ \\
\end{tabular}} & \multicolumn{6}{|c|}{$\mathrm{N}$ treatments } \\
\hline & & \multicolumn{3}{|c|}{2007} & \multicolumn{3}{|c|}{2008} \\
\hline & & $\mathbf{N}_{1}$ & $\mathrm{~N}_{2}$ & $\mathbf{N}_{3}$ & $\mathbf{N}_{1}$ & $\mathrm{~N}_{2}$ & $\mathbf{N}_{3}$ \\
\hline & & $21.13 f$ & $71.65 d$ & $71.65 c$ & $24.00 f$ & $46.50 \mathrm{~d}$ & $71.80 \mathrm{c}$ \\
\hline & $\mathrm{K}_{2}$ & 41.81de & $108.69 c$ & 108.69a & $43.80 \mathrm{~d}$ & $73.00 \mathrm{c}$ & 109.90a \\
\hline & $\mathbf{K}_{3}$ & $36.59 \mathrm{e}$ & $69.11 \mathrm{c}$ & $99.26 b$ & $38.30 \mathrm{e}$ & $71.26 c$ & $101.81 b$ \\
\hline & $\mathrm{K}_{4}$ & $36.03 e$ & $69.94 c$ & $101.33 b$ & $38.96 \mathrm{e}$ & $70.50 \mathrm{c}$ & 103.00b \\
\hline & $\mathrm{K}_{5}$ & $36.16 \mathrm{e}$ & $69.06 c$ & $102.37 b$ & $37.35 \mathrm{e}$ & $72.00 \mathrm{c}$ & 103.00b \\
\hline & $\mathbf{K}_{6}$ & $21.70 f$ & $44.54 d$ & $71.97 \mathrm{c}$ & $23.40 f$ & $45.92 d$ & $72.60 \mathrm{c}$ \\
\hline & $\mathrm{K}_{7}$ & $21.52 f$ & $44.11 d$ & $72.38 \mathrm{c}$ & $23.90 f$ & $46.33 d$ & $73.00 c$ \\
\hline & $\mathbf{K}_{8}$ & $19.12 f$ & $43.87 d$ & $72.23 c$ & $24.40 f$ & $46.22 d$ & $73.30 c$ \\
\hline \multirow{11}{*}{ 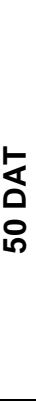 } & \multirow{3}{*}{ K-treatments } & \multicolumn{6}{|c|}{$\mathrm{N}$ - treatments } \\
\hline & & \multicolumn{3}{|c|}{2007} & \multicolumn{3}{|c|}{2008} \\
\hline & & $\mathbf{N}_{1}$ & $\mathrm{~N}_{2}$ & $\mathbf{N}_{3}$ & $\mathbf{N}_{1}$ & $\mathbf{N}_{2}$ & $\mathbf{N}_{3}$ \\
\hline & $\mathrm{K}_{1}$ & $45.15 j$ & $60.90 \mathrm{~h}$ & $99.80 \mathrm{~d}$ & $46.25 \mathrm{k}$ & $61.32 i$ & $100.21 d$ \\
\hline & $\mathrm{K}_{2}$ & $68.50 \mathrm{~g}$ & $101.00 d$ & $136.00 a$ & $69.10 \mathrm{~g}$ & $102.12 d$ & $136.50 \mathrm{a}$ \\
\hline & $\mathbf{K}_{3}$ & $66.60 \mathrm{~g}$ & $100.00 \mathrm{~d}$ & $136.91 a$ & $67.20 \mathrm{gh}$ & $101.00 d$ & $135.00 a$ \\
\hline & $\mathrm{K}_{4}$ & $59.10 \mathrm{~h}$ & $90.00 \mathrm{e}$ & $125.00 b$ & $60.40 \mathrm{i}$ & $91.70 \mathrm{e}$ & $126.30 \mathrm{~b}$ \\
\hline & $\mathrm{K}_{5}$ & $67.30 \mathrm{~g}$ & $101.20 d$ & $136.06 a$ & $69.33 \mathrm{~g}$ & $103.60 d$ & $136.90 a$ \\
\hline & $\mathrm{K}_{6}$ & $54.30 \mathrm{i}$ & $81.30 f$ & $110.25 c$ & $55.10 \mathrm{j}$ & $82.00 f$ & $111.33 c$ \\
\hline & $\mathbf{K}_{7}$ & $46.00 \mathrm{j}$ & $61.66 \mathrm{~h}$ & $100.02 d$ & $46.15 \mathrm{k}$ & 63.20hi & $102.10 d$ \\
\hline & $\mathrm{K}_{8}$ & $53.66 \mathrm{i}$ & $82.26 f$ & $111.00 \mathrm{c}$ & $55.00 \mathrm{j}$ & $88.95 e$ & $112.00 \mathrm{c}$ \\
\hline & \multirow{3}{*}{ K-treatments } & \multicolumn{6}{|c|}{ treatments } \\
\hline & & \multicolumn{3}{|c|}{2007} & \multicolumn{3}{|c|}{2008} \\
\hline & & $\mathbf{N}_{1}$ & $\mathbf{N}_{2}$ & $\mathbf{N}_{3}$ & $\mathbf{N}_{1}$ & $\mathrm{~N}_{2}$ & $\mathbf{N}_{3}$ \\
\hline \multirow{8}{*}{ 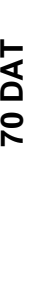 } & $\mathrm{K}_{1}$ & $72.30 p$ & $99.00 \mathrm{k}$ & $135.00 f$ & $73.80 r$ & $100.43 m$ & $134.01 \mathrm{hi}$ \\
\hline & $\mathrm{K}_{2}$ & $98.00 \mathrm{k}$ & $136.00 f$ & $171.00 b$ & $99.03 \mathrm{~m}$ & $138.00 \mathrm{gh}$ & 171.00b \\
\hline & $K_{3}$ & 90.801 & $129.20 \mathrm{~g}$ & $168.00 \mathrm{bc}$ & $91.06 n$ & $131.50 \mathrm{l}$ & 167.10bc \\
\hline & $\mathrm{K}_{4}$ & 87.30lm & 121.25h & 165.30cd & 88.21 no & 123.10j & $165.00 \mathrm{c}$ \\
\hline & $\mathrm{K}_{5}$ & $99.40 \mathrm{k}$ & $141.32 \mathrm{e}$ & $178.10 a$ & $100.90 \mathrm{~m}$ & 143.00ef & $177.90 \mathrm{a}$ \\
\hline & $\mathrm{K}_{6}$ & 81.60no & $116.00 \mathrm{i}$ & $145.15 \mathrm{e}$ & $82.40 \mathrm{pq}$ & $118.20 \mathrm{k}$ & $144.00 \mathrm{e}$ \\
\hline & $\mathbf{K}_{7}$ & 78.500 & $108.00 \mathrm{j}$ & 140.10ef & $78.90 q$ & $110.00 \mathrm{~L}$ & $139.20 f g$ \\
\hline & $\mathbf{K}_{8}$ & $85.20 \mathrm{mn}$ & 120.00hi & $162.00 d$ & $85.00 \mathrm{op}$ & 112.00jk & $160.00 \mathrm{~d}$ \\
\hline
\end{tabular}


El-Hamdi, Kh. H. et al.

Table 13: Potassium uptake $\left(\mathrm{Kg} \mathrm{fed}^{-1}\right)$ at 95 DAT (grain and straw) as affected by interaction between different nitrogen levels and methods of potassium application during 2007 and 2008 seasons.

\begin{tabular}{|c|c|c|c|c|c|c|c|}
\hline & \multirow{3}{*}{ K-treatments } & \multicolumn{6}{|c|}{ N- treatments } \\
\hline & & & 2007 & & & 2008 & \\
\hline & & $\mathbf{N}_{1}$ & $\mathbf{N}_{2}$ & $\mathbf{N}_{3}$ & $\mathbf{N}_{1}$ & $\mathbf{N}_{2}$ & $\mathbf{N}_{3}$ \\
\hline \multirow{8}{*}{ 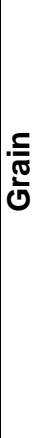 } & $\overline{K_{1}}$ & $8.05 j$ & 14.09hi & 20.00 & 8.501 & 14.66ijk & 20.50d-h \\
\hline & $\mathrm{K}_{2}$ & 14.59hi & 20.83def & 25.62 & $15.80 \mathrm{ij}$ & $21.00 c-h$ & $24.90 \mathrm{~b}$ \\
\hline & $\mathrm{K}_{3}$ & 13.63hi & 20.00efg & 24.00 & $14.00 \mathrm{ijk}$ & $20.40 e-h$ & $23.66 \mathrm{bc}$ \\
\hline & $\mathrm{K}_{\mathbf{4}}$ & 13.25hi & 19.90efg & 23.03 & $13.06 \mathrm{k}$ & $21.09 c-h$ & $23.36 b c$ \\
\hline & $\mathrm{K}_{5}$ & $15.40 \mathrm{~h}$ & 21.80 cde & 27.60 & $16.00 \mathrm{i}$ & 22.90be & $27.92 a$ \\
\hline & $\mathrm{K}_{6}$ & 12.80hi & $18.50 \mathrm{fg}$ & 22.90 & $12.50 \mathrm{k}$ & 19.30gh & $22.46 b f$ \\
\hline & $\mathrm{K}_{7}$ & $12.00 \mathrm{i}$ & $18.00 \mathrm{~g}$ & 20.66 & $12.00 \mathrm{k}$ & $18.99 \mathrm{~h}$ & $22.00 \mathrm{c}-\mathrm{g}$ \\
\hline & $\mathrm{K}_{8}$ & 12.90hi & 19.40efg & 24.00 & 13.30jk & $20.30 f g h$ & 23.15bcd \\
\hline \multirow{3}{*}{\multicolumn{2}{|c|}{ K-treatments }} & \multicolumn{6}{|c|}{$\mathrm{N}$ - treatments $\left(\mathrm{kg} \mathrm{fed}^{-1}\right)$} \\
\hline & & & 2007 & & & 2008 & \\
\hline & & $\mathbf{N}_{1}$ & $\mathbf{N}_{2}$ & $\mathbf{N}_{3}$ & $\mathbf{N}_{1}$ & $\mathrm{~N}_{2}$ & $\mathbf{N}_{3}$ \\
\hline \multirow{8}{*}{ 离 } & $\overline{K_{1}}$ & $62.60 \mathrm{~m}$ & $90.00 \mathrm{i}$ & 104.90ef & $64.00 n$ & 93.00j & $107.75 \mathrm{~g}$ \\
\hline & $\mathrm{K}_{2}$ & 79.95jk & $106.00 \mathrm{e}$ & $117.20 \mathrm{~b}$ & $82.50 \mathrm{k}$ & $110.33 f$ & $121.20 b$ \\
\hline & $\mathrm{K}_{3}$ & 78.00k & 103.50ef & $116.50 \mathrm{~b}$ & $76.25 \mathrm{~lm}$ & $106.25 \mathrm{gh}$ & $120.00 \mathrm{~b}$ \\
\hline & $\mathrm{K}_{\mathbf{4}}$ & 75.001 & $103.00 \mathrm{fg}$ & $112.00 c$ & $75.00 \mathrm{~m}$ & $107.75 \mathrm{~g}$ & $116.80 c$ \\
\hline & $\mathrm{K}_{5}$ & $81.50 \mathrm{j}$ & $111.20 \mathrm{~cd}$ & $122.00 a$ & $83.15 \mathrm{k}$ & 114.00de & $124.80 a$ \\
\hline & $\overline{K_{6}}$ & 73.60l & $100.60 \mathrm{gh}$ & $109.00 d$ & $76.30 \mathrm{~lm}$ & 104.00h & $115.00 \mathrm{cds}$ \\
\hline & $\mathrm{K}_{7}$ & 73.50I & $100.00 \mathrm{~h}$ & $108.90 d$ & $75.50 \mathrm{~m}$ & $99.33 i$ & $113.00 \mathrm{e}$ \\
\hline & $\mathrm{K}_{8}$ & 75.001 & $102.50 \mathrm{fd}$ & $110.00 \mathrm{~cd}$ & $78.70 \mid$ & $105.00 \mathrm{~h}$ & $116.00 c$ \\
\hline
\end{tabular}

3. Effect of nitrogen and potassium fertilization on grain yield

3- Grain Yield:

Grain yield of hybrid rice as affected by the application of nitrogen levels and methods of potassium application in 2007 and 2008 seasons are presented in Tables 14 and 15.

Data indicate that there was a significant difference in grain yield due to nitrogen fertilizer application. Data show that significant increase in grain yield as nitrogen levels increased from 0 up to $92 \mathrm{Kg} \mathrm{N}$ fed $^{-1}$ in both seasons of study. The increased grain yield owing to $\mathrm{N}$ fertilization was attributed directly by significantly improvement in the yield attributed like number of panicles per $\mathrm{m}^{2}$, panicle weight and filled grains percentage. The results confirm the findings of Badasubramanian (2002), Samrathlal et al. (2003), Singh et al. (2004), Zaheen et al. (2006) and Sikdar et al. (2008). 
Table14: Grain yield $\left(t_{\text {fed }}{ }^{-1}\right)$ as affected by different nitrogen levels and methods of potassium application.

\begin{tabular}{|c|c|c|}
\hline \multirow{2}{*}{ Treatment } & \multicolumn{2}{|c|}{ Grain } \\
\cline { 2 - 3 } & $\mathbf{2 0 0 7}$ & $\mathbf{2 0 0 8}$ \\
\hline $\mathbf{N}$ - treatments & & $3.085 \mathrm{c}$ \\
\hline $\mathbf{N}_{\mathbf{1}}$ & $3.044 \mathrm{c}$ & $4.952 \mathrm{~b}$ \\
\hline $\mathbf{N}_{2}$ & $4.805 \mathrm{~b}$ & $5.378 \mathrm{a}$ \\
\hline $\mathbf{N}_{3}$ & $5.290 \mathrm{a}$ & \\
\hline $\mathbf{K}-$ treatments & & $3.908 \mathrm{f}$ \\
\hline $\mathbf{K}_{1}$ & $3.752 \mathrm{e}$ & $4.737 \mathrm{~b}$ \\
\hline $\mathbf{K}_{2}$ & $4.661 \mathrm{~b}$ & $4.555 \mathrm{c}$ \\
\hline $\mathbf{K}_{3}$ & $4.517 \mathrm{c}$ & $4.437 \mathrm{~d}$ \\
\hline $\mathbf{K}_{4}$ & $4.442 \mathrm{c}$ & $5.137 \mathrm{a}$ \\
\hline $\mathbf{K}_{5}$ & $4.925 \mathrm{a}$ & $4.340 \mathrm{de}$ \\
\hline $\mathbf{K}_{6}$ & $4.215 \mathrm{~d}$ & $4.250 \mathrm{e}$ \\
\hline $\mathbf{K}_{\mathbf{7}}$ & $4.167 \mathrm{~d}$ & $4.412 \mathrm{~d}$ \\
\hline $\mathbf{K}_{8}$ & $4.360 \mathrm{c}$ & $\mathrm{c}$ \\
\hline
\end{tabular}

Where: DAT: days after transplanting, $N_{1}: 0 \mathrm{~kg} \mathrm{~N}$ fed-1., $\mathrm{N}_{2}: 69 \mathrm{~kg} \mathrm{~N}$ fed-1, $\mathrm{N}_{3}: 92 \mathrm{~kg} \mathrm{~N}$ fed1., $K_{1}$ : Zero potassium., $K_{2}: 50 \mathrm{~kg}$ as basal., $K_{3}: 25 \mathrm{~kg}$ as basal $+2 \%$ at $P . I, K_{4}: 25$ $\mathrm{kg}$ as basal $+2 \%$ at $F, K_{5}: 25 \mathrm{~kg}$ as basal $+2 \%$ at $P . I$ and $F ., K_{6}: 2 \%$ at P.I., $K_{7}: 2 \%$ at $F_{.}, K_{8}: 2 \%$ at P.I and F.

Grain yield of hybrid rice as affected by the methods of potassium in both seasons are presented in Table 14. Data indicate that grain yield was significantly affected by method of potassium application in both seasons. Half dose of potassium before transplanting ( $25 \mathrm{~kg}$ potassium sulphate) + spray with $2 \% \mathrm{~K}_{2} \mathrm{O}(4 \mathrm{~kg}$ potassium sulphate) at panicle initiation stage + spray with $2 \% \mathrm{~K}_{2} \mathrm{O}$ (4 $\mathrm{kg}$ potassium sulphate) at flowering stage produced significantly higher grain yield compared with the other treatments. This might be owing to the continuous supply of potassium to the crop during cropgrowth period which is more beneficial and increased translocation of carbohydrates form stems, leaf sheathes and other storage organs to grains, leading to high sink capacity and, subsequently, higher grain yield. Similar trend was obtained by Janardan et al. (2000) and Zaratin et al. (2004).

Data document in Table 15 reveal that the interaction between nitrogen levels and methods of potassium application had a significant effect on grain yield in 2007 and 2008 seasons. Application of nitrogen at the rate of $92 \mathrm{Kg} \mathrm{N}$ fed $^{-1}$ in combined with application half dose of recommended amount potassium before transplanting ( $25 \mathrm{~kg}$ potassium sulphate) + spray with $2 \%$ $\mathrm{K}_{2} \mathrm{O}\left(4 \mathrm{~kg}\right.$ potassium sulphate) at panicle initiation stage + spray with $2 \% \mathrm{~K}_{2} \mathrm{O}$ (4 $\mathrm{kg}$ potassium sulphate) at flowering stage were superior to other treatments While, the minimum values were obtained when nitrogen and potassium did not applied. The increase in grain yield is attributed mainly to the increase in most yield components, i.e. number of panicle per $\mathrm{m}^{2}$, filled grains percentage and 1000-grain weight. Similar trend was obtained by Manivannan et al. (2005), Dwived et al. (2006) and Bahmaniar and Ranjbar (2007). 
El-Hamdi, Kh. H. et al.

Table 15: Grain yield $\left(t\right.$ fed $\left.^{-1}\right)$ as affected by the interaction between nitrogen levels and methodsof potassium application during 2007 and 2008 seasons.

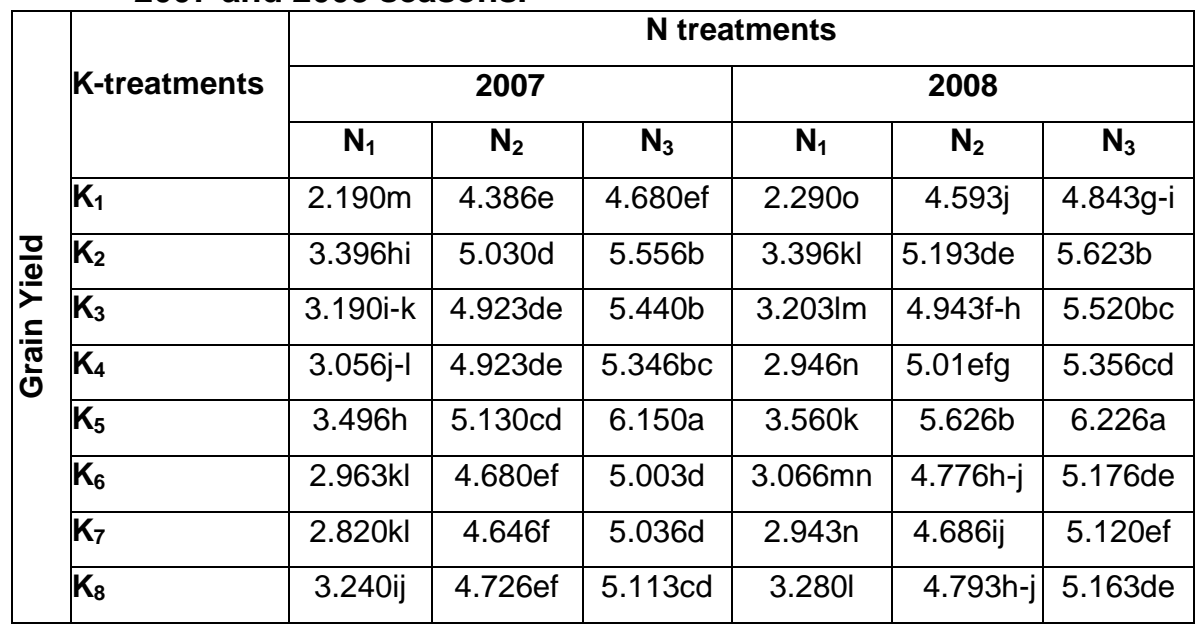

Where: DAT: days after transplanting, $\mathrm{N}_{1}: 0 \mathrm{~kg} \mathrm{~N}$ fed-1., $\mathrm{N}_{2}: 69 \mathrm{~kg} \mathrm{~N}$ fed-1, $\mathrm{N}_{3}: 92 \mathrm{~kg} \mathrm{~N}$ fed1., $K_{1}$ : Zero potassium., $K_{2}: 50 \mathrm{~kg}$ as basal., $K_{3}: 25 \mathrm{~kg}$ as basal $+2 \%$ at $P . \mathrm{l}, K_{4}: 25$ $\mathrm{kg}$ as basal $+2 \%$ at $F, K_{5}: 25 \mathrm{~kg}$ as basal $+2 \%$ at P.I and F., $K_{6}: 2 \%$ at P.I., $K_{7}: 2 \%$ at F., $K_{8}: 2 \%$ at P.I and F.

\section{CONCLUSION}

From this study, data obtained could be recommended that applying of nitrogen fertilizer at the rate of $92 \mathrm{Kg} \mathrm{N} \mathrm{fed}^{-1}$ along with potassium at basal application as a half dose (25 kg potassium sulphate) + spray with $2 \% \mathrm{~K}_{2} \mathrm{O}(4$ $\mathrm{kg}$ potassium sulphate) at panicle initiation and flowering growth stages produced the peak values of both availability and uptake of nitrogen and potassium at growth periods as well as grain rice yield.

\section{REFERENCES}

Ali, A.; M.S. Zia; F. Hussain; M. Slim and A. Shahzad (2005). Efficacy of different methods of potassium fertilizer application on paddy yield, $\mathrm{K}$ uptake and agronomic efficiency. Pak. J. Agric. Sci., 42 (1-2): 27-32.

Akhtar, M.S. (1989). Soil minerology and potassium quantity / intensity relation in the alluvial soils from Pakistan. Dept. Soil and Crop Sciences, Taxas A \& M University, Ph. D. Dissertation.

Arshad, M. and A. Akram. (1999). Soil fertility problems in central Rachna Doab, Pak. J. Bio. Sci., 2 (4): 1355 - 1357.

Arivazhagan, K. and M. Ravichandran (2005). Interaction effect of nitrogen and potassium on yield and yield attributes in rice cv. IR20. Advances Plant Sci., 18(1):425-427.

Badasubramanian, R. (2002). Response of hybrid rice to levels and time of application of nitrogen. Indian J. Agron., 47(2): 203-206. 
Bahmaniar, M.A. and G.A. Ranjbar (2007). Response of rice cultivars to rates of nitrogen and potassium application in field and pot conditions. J. Biological Sci. 10(9)1430-1437.

Bouabid, R., M. Badraoui and P. R. Bloom. (1991). Potassium fixation and charge characteristics of soil clays. Soil Sci. Soc. Am. J., 55: 1493 1498.

Chapman, H. D. and P. F. Parrt (1961). Method of Analysis for Soils, Plant and Water. Univ. of California, USA.

Cottenie, A.M. Verloo; L. Kiekens, G. Velghe and R. Camerlynch (1982). Chemical analysis of plants and soils. Laboratory of Analytical Agro. Chemistry state University, Ghent Belgium.

De Datta, S.K. (1981). "Principles and practices of rice production". AWileyInter Science Publication New York.

Devendra, S.; V. Singh and R. Singh (1999). Response of rice to nitrogen and potassium levels in alluvial soil. J. Indian, Agron. 15 (1/4):88 - 92.

Dwivedi, A.P.; R.S. Dixit and G.R. Singh (2006). Effect of nitrogen, phosphorus and potassium levels on growth, yield and quality of hybrid rice (Oryza Sativa). Indian J. Agron., 43(1) : 64-66.

Hafez, A.A.R. and D.S. Mikkelson (1981). Colorimetric determination of nitrogen for evaluating the nutrition status of rice. Soil Sci. and Plant Analy. 12(1):61-69.

Hammad, S.A.; (1995). Pollution of leaching water and some nutrients available as a parameter of effeiciency of biological and inorganic sources under submergence condition. J. Agric. Sci. Mansoura Univ, 20(4): $1915-1931$.

Janardan - Singh; H.L. Sharma and C.M. Singh (2000). Effect of levels and phases of potassium application on growth and yield of rice and wheat. J. Indian Res., 16 (1/4):35-40. (C.F. Rice Abs.)

Krishnakumar, S.; R. Nagarajan; S.K. Natarajan; S Jawahar and J.B. Pandian (2005). NPK fertilizers for hybrid rice (oryza sativa) productivity in Alfisols of sothern Districts of Tamil Nadu. Asian J. Plant Sci., 4(6):574576.

Maiti, S.; N. Naleshwar and S. Pal (2003). Response of high yielding and hybrid rice to varied levels of nitrogen nutrition. Environment and Ecology, 21(2): 296-300.

Manivannan, K.; S. Natarajan; M. Ganapathy and K. Arivazhagan (2005). Effect of different levels of nitrogen and potassium on growth, nutrient uptake and yield of rice hybrids. J. Ecobiology, Annamalai Univ., 17(6): 593-595.

Meena, S.L.; S. Surenda and Y.S. Shivay (2002). Response of hybrid rice to nitrogen and potassium application. Indian J. of Agron., 47(2): 207-211.

Page, A.L.; R.H. Miller and D.R. Keeney (1982). Method of Soil Analysis part 2. Amer. Sco. Agric. Inc. Madison.

Pattanayak, S.K.; S.K. Mukhi and K. Majumdar (2008). Potassium unlocks the potential for hybrid rice. Better Crops, 92(2).

Samrathlal, M.; S. Surendra and Y.S.Shivay (2003). Response of hybrid rice to nitrogen and potassium application in sandy clay-loam soils. Indian J. Agric. Sci., 73 (1): 8-11. 


\section{El-Hamdi, Kh. H. et al.}

Sikdar, M.S.I.; M.M. Rahman; M.S. Islam; M.S. Yeasmin and M.M. Akhter (2008). Effect of nitrogen level on aromatic rice varieties and soil fertility status. Int. J. Sustain. Crop Prod., 3(3) 49-54.

Singh, T.; Y.S. Shivay and S. Singh (2004). Effect of date of transplanting and nitrogen on productivity and nitrogen use indices in hybrid and non-hybrid aromatic rice. Indian Agric. Res. Institute, 52(3): 245-252.

Slaton, N.A.; D. Dunn and B. Pugh (2004). Potassium nutrition flood-irrigated rice. Better Crops, 88 (3).

Suresh, S.; P.P. Ramaswami and R.R. Kennedy (1995). Effect of nitrogen sources on the availability of inorganic nitrogen forms in sandy clay wetland rice soil. Madras Agricultural Journal, 82 (6.7.8): 472-476.

Thakur D.S.; S. R. Patel and Nageshwarlal, (1999). Effect of split application of potassium with FYM on rice (Oryza Sativa). Indian J. Agron., 44 (2): $301-304$.

Thippeswamy, H.M.; B.G. Shivakumar and S.S. Balloli (2000). Potassium transformation studies in lowland rice (oryza sativa) as influenced by levels and time of K application. J. Indian Soci. Soil Sci., 16(4):7-11.

Tisdale, S.L.; W.L.Nelson and J.D.Beation (1985). Soil fertility and fertilizers (fourth edition). Macmillan Publishing Company, 866 Third Avenue New York.

Wang, Q.S.; R.H. Zhen; Y.F. Ding; J.Z. Jun ND w. Cao (2004). Effects of potassium fertilizer application rates on plant potassium accumulation and grain quality of japonica rice. China Scientia Agric. Sinica, 37(10)1444-1450.

Wihardjaka, A.G.; G.J. Kirk and C.P. Mamank (1999). Potassium balances in rain-fed lowland rice on a light textured soil. Field Crops Res., 64: 237-247.

Young, X. E.; L. H. Krik and A. Dobbermann (2005). Room - induced changes of potassium in the rhizosphere of lowland rice. Communications in soils. In Soil Sci. and Plant Analysis, 36 (13): 19471963.

Zaheen, M; H.A. Tahir; M.S. Ehsan; A.I. Rana; M.M. Ashraf and A. Mushtaq (2006). Effect of nitrogen levels on yield and yield components of basmati 2000. J. Agric. Res., 44(2).

Zaratin, C.; S.A. Souza; A.C. Pantano; M.E. Sa and S. Buzetti (2004). Effects of four potassium doses on six sprinker irrigated rice upland cultivars .I. yield and yield components. Cientifica Jaboticabal, 32 (2);115-120.

Zhang-Hong, C.; X.Q. Wang; Q.G. Dai; Z.Y. Huo and Xu-Ke (2003). Effects of $\mathrm{N}$ application rate on the yield, quality and nitrogen uptake of hybrid rice variety Liangyoupeijiu. Chinese, Scientia Agricultura Sinica, 36(7):80-806. 
تيسر وامتصاص النيتروجين والبوتاسيوم تحت التسميذ بالأسمدة الغير عضوية فى أراضى الأرز المغمورة

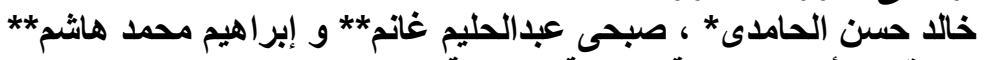

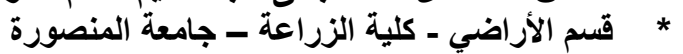

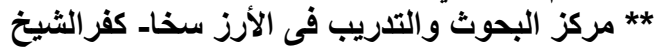

أجريت تجربتان حقليتان فى مزرعة مركز البحوث و التدريب فى الأرز - سخا كفر الثيخ

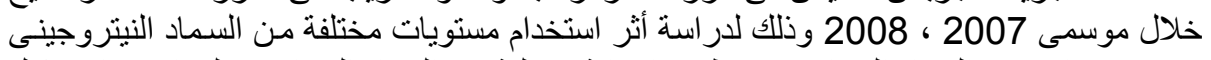

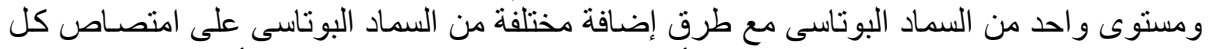

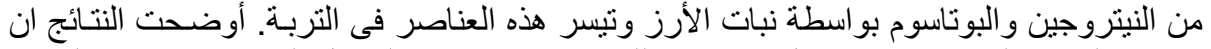

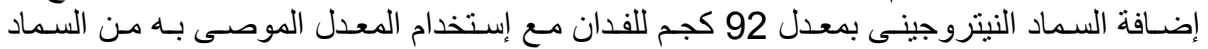

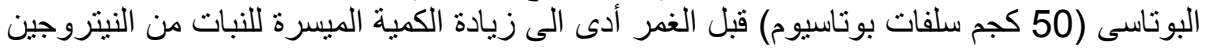

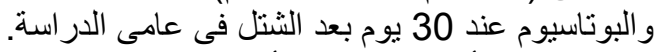

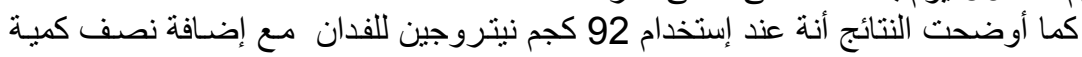

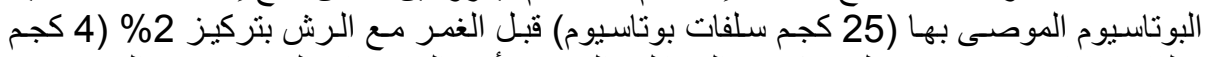

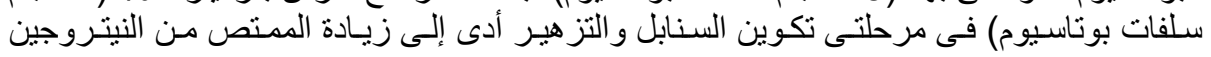

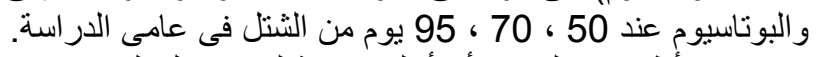

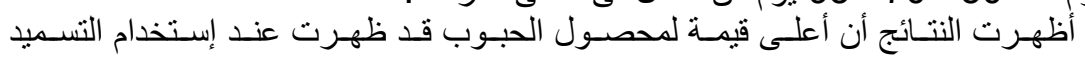

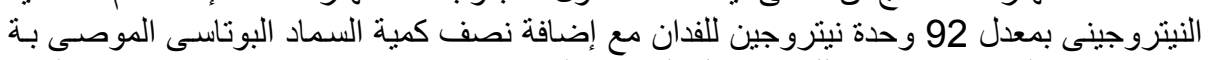

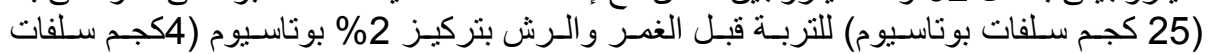
بوتاسيوم) فى مرحلتى تكوين السنابل و التز هير.

كلية الزراعة - جامعة المنصورة كلية الزراعة - جامعة عين شمس الزعس

$$
\begin{aligned}
& \text { قام بتحكيم البحث } \\
& \text { أ.د / أحمد عبد القادر طه } \\
& \text { أ.د / عيد مرسسى خالد }
\end{aligned}
$$

